\title{
Redes sociales y territorialidad bolichera de sirios y libaneses en el sudeste de Río Negro, Argentina (1900-1950)*
}

\section{Resumen}

En este artículo proponemos un análisis sobre las relaciones establecidas por los inmigrantes sirios y libaneses en el sudeste del Territorio Nacional de Río Negro en las primeras décadas del siglo XX. El uso de la metodología de Análisis de Redes Sociales (ARS) nos permitió discutir las caracterizaciones estereotipadas de estos sujetos históricos para reinstalar su complejidad y la multiplicidad de estrategias, individuales y colectivas, con el propósito de avanzar en la reconstrucción de una cartografía que habilite la representación gráfica de las redes de familiarización/ alianza. Espacialmente, nos circunscribimos al sudeste de Río Negro, y dentro de este territorio prestamos una mayor atención a la localidad de Valcheta y sus zonas de influencia para determinar la posición y la movilidad de los actores a partir del estudio de una amplia variedad de fuentes producidas mayormente por el estado.

Palabras clave: Patagonia, bolicheros, sirios, libaneses, redes sociales, territorialidad.

Referencia para citar este artículo: CHÁVEZ, Matías Rodrigo y SOURROUILLE, Marcos (2016). "Redes sociales y territorialidad bolichera de sirios y libaneses en el sudeste de Río Negro, Argentina (1900-1950)", en Anuario de Historia Regional y de las Fronteras, 21 (1). pp. 159-181.

Matías Rodrigo Chávez: Actualmente, cursa el Doctorado en Historia (UNCPBA). Becario doctoral Instituto Patagónico de Ciencias Sociales y Humanas CENPAT-CONICET. Licenciado en Historia (UNPSJB). Correo electrónico: chavez@cenpat-conicet.gob.ar.

Marcos Sourrouille: Actualmente, cursa el Doctorado en Filosofía y Letras (UBA). Becario doctoral (ANPCyT) Instituto Patagónico de Ciencias Sociales y Humanas CENPAT-CONICET. Profesor en Historia (UBA). Correo electrónico: soumarcos48@gmail.com.

* Artículo de investigación científica derivado parcialmente de la tesis inédita para obtener el título de Licenciado en Historia, de Matías Chávez 


\title{
Social Networks and Seiner Territoriality of Syrians and Lebanese in the Rio Negro Southwest region, Argentina (1900-1950)
}

\begin{abstract}
The authors of this article analyze the relation of Lebanese and Syrian immigrants at the Rio Negro National Southwest region during the first decades of the $20^{\text {th }}$ century. The utilization of a Social Network Analysis methodology (SNA) contributed to the analysis of stereotyped characterization of these historical individuals in order to redefine the complexity and multiplicity of strategies, both at individual and collective levels. The purpose is to advance toward the reconstruction of a cartography that makes graphic representations of the family / alliance relation networks. At space level, the study focuses on the southwest area of Rio Negro and, within this territory, we concentrated in the town of Valcheta and its area of influence in order to determine the position and mobility of actors based on a broad variety of sources produced mainly by the State.
\end{abstract}

Keywords: Patagonia, bolicheros, Syrians, Lebanese, social networks, territorialuty.

\section{Redes sociais e territorialidade baladeira de sírios e libaneses no sudeste de Rio Negro, Argentina (1900-1950)}

\section{Resumo}

Neste artigo propomos uma análise sobre as relações estabelecidas pelos imigrantes sírios e libaneses no sudeste do Território Nacional de Rio Negro nas primeiras décadas do século XX. O uso da metodologia de Análise de Redes Sociais (ARS) nos permitiu discutir as características estereotipadas de estes sujeitos históricos para reinstalar sua complexidade e a multiplicidade de estratégias, individuais e coletivas, com o propósito de avançar na reconstrução de uma cartografia que habilite a representação gráfica das redes de familiarização/aliança. Especialmente, nos circunscrevemos ao sudeste de Rio Negro, e dentro deste território prestamos maior atenção a localidade de Valcheta e suas zonas de influência, para determinar a posição e mobilidade dos atores a partir do estudo de uma ampla variedade de fontes produzidas principalmente pelo Estado.

Palavras-chave: Patagonia, baladeiros, sírios, libaneses, redes sociais, territorialidade. 


\section{Introducción}

Nos interesa discutir las relaciones establecidas y la territorialidad construida por los inmigrantes sirios y libaneses en el sudeste de Río Negro ${ }^{1}$ dentro de la región patagónica durante la primera mitad del siglo XX, con el fin de identificar sus redes de solidaridad e indagar sobre la configuración de sus zonas de influencia. Consideramos oportuno precisar que en la Patagonia se denomina como boliches a los comercios de ramos generales, ubicados generalmente en zonas rurales. El boliche no solo fue un eje articulador y distribuidor de mercancías y frutos del país, sino que también fue un espacio de socialización y un lugar de tránsito, por lo cual nos interesa el boliche más como un nodo de sociabilidad que como un centro comercial. Específicamente, nos centramos en los bolicheros como líderes de esos espacios comerciales y de sus redes derivadas.

En la búsqueda por identificar a los inmigrantes sirios y libaneses, y los bolicheros de ese origen, analizamos diferentes tipologías documentales producidas por el estado. Utilizamos informes policiales, inspecciones de la Dirección General de Tierras en San Antonio y la Colonia Valcheta con sus zonas de influencia I y II y el Registro Único de Comercios disponibles en el Archivo Histórico de la Provincia de Río Negro (AHPRN). Asimismo, consideraremos la documentación producida por el Registro Civil de Valcheta, los documentos personales de sirios y libaneses y los artículos periodísticos sobre la colectividad, expuestos en el Archivo del Museo Regional María Inés Koop; además de escritos resguardados por los descendientes en el ámbito familiar.

En cuanto a la periodización propuesta, tenemos en cuenta que desde los primeros años del siglo XX comenzaron a asentarse en el sudeste de Río Negro inmigrantes sirios y libaneses, algunos de los cuales comenzaron a operar comercialmente, dando inicio a un lento proceso de acumulación. Su ciclo de ascenso económico fue mayoritariamente de mercachifles a bolicheros y habría durado hasta 1930 para luego empezar una gradual decadencia o reconversión con posterioridad a 1940. Parte de nuestros objetivos es matizar la supuesta desaparición del boliche en esa coyuntura, inclinándonos a pensar el proceso más como un reacomodamiento comercial que como una extinción absoluta, lo que será planteado en la última parte de este artículo a partir de su relevancia al momento de discutir la pervivencia o la rearticulación de las redes sociales.

\footnotetext{
${ }^{1}$ El Territorio Nacional de Río Negro fue creado como una unidad administrativa en 1884 por la Ley n. ${ }^{\circ}$ 1532. Desde 1882 estas tierras formaban parte de la Gobernación de la Patagonia. Las campañas militares de conquista de la Patagonia septentrional y central se desarrollaron entre fines de la década de 1870 y mediados de la de 1880, lo que implicó el quiebre de la autonomía política y -en un proceso más lento y gradual- del control territorial de las poblaciones mapuches y tehuelches que habitan la región. Como señala Iuorno, la provincialización de Río Negro fue un proceso complejo: si bien la provincia se creó en 1955 a través de la Ley n. ${ }^{\circ}$ 14408, las primeras elecciones populares se realizarían en 1958, luego de un interregno conflictivo: Iuorno, Graciela. "La provincialización de Río Negro. Interregno y conflictos de intereses nacionales y locales", en Ruffini, Martha y Masera, Ricardo (coords.), Horizontes en perspectiva. Contribuciones para la Historia de Río Negro 1884-1955 (Viedma: Fundación Ameghino, 2007), pp. 389-405.
} 
En relación a los alcances del espacio geográfico enunciado, el sudeste de Río Negro dentro de la Patagonia, trabajaremos sobre el primer tramo de la denominada línea sur, usando como eje a la localidad de Valcheta, con particular atención en los parajes ${ }^{2}$ de su zona de influencia, aunque contemplando a las localidades adyacentes como San Antonio y Sierra Grande.

Mapa 1. Parajes de residencia de sirios y libaneses (1900-1940).

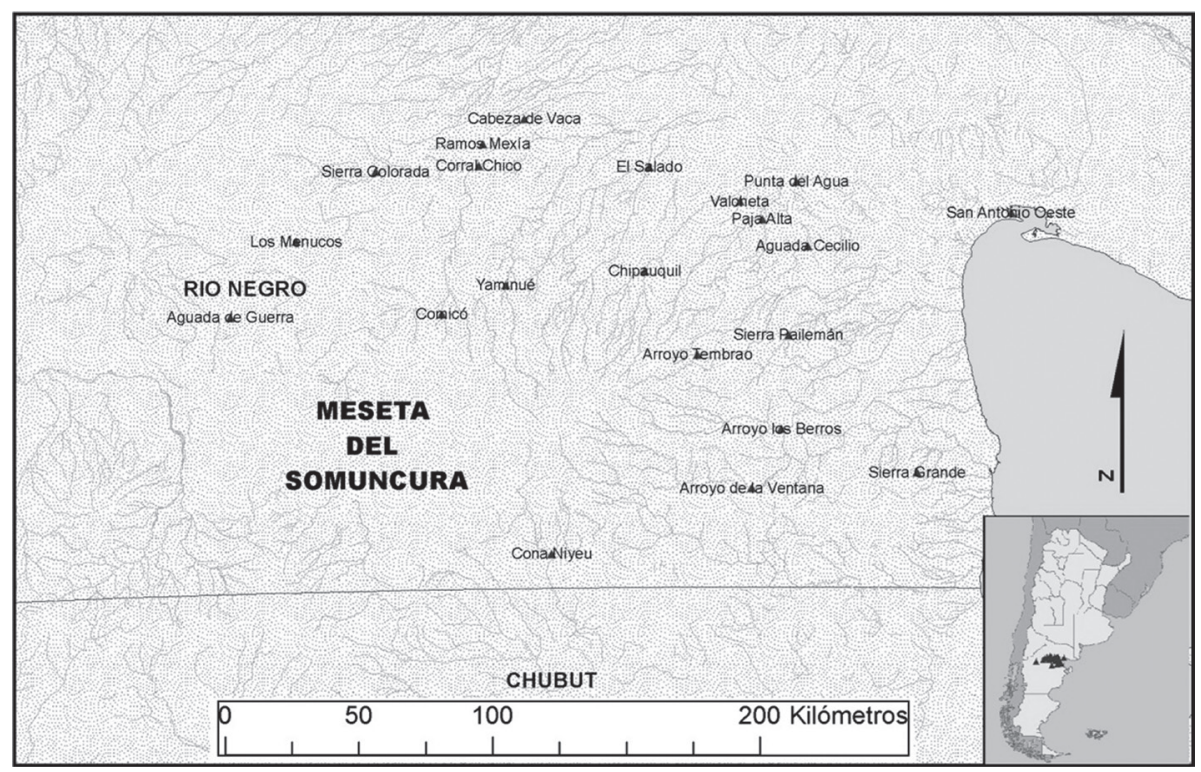

Fuente: elaboración de los autores, a partir de los datos relevados en el Archivo Histórico de la Provincia de Río Negro y la delegación del Registro Civil en Valcheta.

De los relevamientos realizados surge la idea de que este espacio mantenía una dinámica propia y parcialmente diferenciada de otras subregiones del interior patagónico septentrional. Los parajes sobre los que se establecieron los comerciantes sirios y libaneses tendieron a rodear la meseta del Somuncurá para formar un contorno mercantil en las márgenes de la formación mesetaria, dicha región se caracterizaba por una bajísima densidad demográfica, además de un tipo de clima y de terreno que suponían fuertes dificultades para la actividad humana ${ }^{3}$.

\footnotetext{
${ }^{2}$ Los parajes son espacios geográficos con escasa población, generalmente dispersa, que funcionan como puntos de tránsito, de producción y de comercialización para los actores del interior patagónico.

${ }^{3}$ Boschín y Andrade caracterizan las mesetas centrales del norte de la Patagonia "[...] por la alternancia de mesetas, cerros y lomas bajas que enmarcan bajos y valles". Entre ellas, "[u]na de las unidades geológicas y geomorfológicas más importantes es la meseta de Somuncurá, una planicie de origen volcánico de 25.000 km2 de extensión localizada en el centro - este de las provincias de Río Negro y del Chubut-. Presenta un fuerte gradiente altitudinal que asciende desde los 600 a los 1.400 m s.n.m., producto de sucesivas emisiones volcánicas" (Boschín, María Teresa y Andrade, Analía. "Poblamiento de Patagonia Septentrional Argentina durante el holoceno tardío: paleoambientes e imperativos sociales", en Zephyrus, n. ${ }^{\circ}$ 68, Salamanca, 2011, pp. 41-61). Boschín y del Castillo Bernal han destacado la importancia arqueológica e histórica de la región a propósito del yamnago, en tanto territorio de caza de las sociedades indígenas,
} 
Por último, trabajaremos a partir de una definición social de región ${ }^{4}$, siguiendo las conexiones sociales y las redes mercantiles de los propios agentes y considerando, como lo plantea De Jong, que "[...] la región comienza y termina donde comienza y termina su explicación"5. La región contempla la movilidad de los comerciantes de la zona de Valcheta que no solían extenderse en sus operaciones más allá de Los Menucos hacia el oeste, de Cabeza de Vaca hacia el norte, de San Antonio hacia el este y hacia el sur, podían cruzar el límite de Río Negro y operar en el norte de Chubut.

\section{Análisis de redes sociales establecidas en el sudeste de Río Negro}

A continuación intentaremos precisar las redes de familias, negocios y territorialidad de los inmigrantes y bolicheros sirios y libaneses en el sudeste de Río Negro ${ }^{6}$. Los grafos de redes ${ }^{7}$ muestran diferentes topologías, sujetos y movilidades para el espacio social y el período analizados. El Análisis de Redes Sociales (ARS) se combina en este trabajo con la metodología de archivo y con una interpretación histórica contextual, procedimientos que tienen en común concebir como punto de partida a los documentos y a los testimonios disponibles. Como propone Reynoso:

[... ] la teoría de grafos y el análisis de redes no niegan ni afirman la legitimidad de otras aproximaciones que alegan ser o que acaso sean marginalmente más concretas, si ello pudiera dirimirse de algún modo; simplemente destacan nuevas formas de entendimiento basadas en un conjunto de estilos de abstracción relacional, representación, modelado y generalización entre los muchos que se presienten posibles ${ }^{8}$.

analizando los condicionamientos geográficos que imponía la meseta del Somuncurá. (Boschín, María Teresa y del Castillo Bernal, Florencia. "El Yamnago: del registro histórico al registro arqueológico", en Revista Española de Antropología Americana, n. ${ }^{\circ}$ 36, Madrid, 2005, pp. 99-116).

${ }^{4}$ Bandieri, Susana. "Entre lo micro y lo macro: la historia regional. Síntesis de un experiencia", en Entrepasados, n. ${ }^{\circ}$ 11, Buenos Aires, 1996, pp. 71-100.

${ }^{5}$ De Jong, Gerardo. Introducción al método regional (Neuquén: Lipat-Universidad Nacional del Comahue, 2001), p. 77.

${ }^{6}$ La metodología del ARS fue introducida por Julio Vezub en el campo de la historia social patagónica, al construir una cartografía de redes parentales indígenas, redes políticas y de negocios a partir del corpus de las cartas de Valentín Saygüeque, Vezub, Julio. "Lenguas, territorialidad y etnicidad en la correspondencia de Valentín Saygüeque hacia 1880”, en Intersecciones en Antropología, n. 7, Olavarria, 2006, pp. 287-304. En otro trabajo Salomón Tarquini, Vezub y Escolar proponen la utilización de ARS apoyada en herramientas informáticas y los Sistemas de Información Geográfica (SIG), en su búsqueda por representar la topología de redes de alianzas y parentesco entre los líderes indígenas pampeano-patagónicos durante la segunda mitad del siglo XIX, hasta establecer la cartografía y la territorialidad de las redes sociopolíticas indígenacriollas a través del seguimiento, por ejemplo, de sus intercambios epistolares. Salomón Tarquini, Claudia, Vezub, Julio y Escolar, Diego. "Cartografía de redes sociales en pampa y Patagonia: sistematización de correspondencia de líderes indígenas mediante bases de datos inteligentes", en Primer Congreso Internacional Las poblaciones indígenas de América Latina, Siglos XIX-XXI. Avances, perspectivas y retos, Oaxaca, 2013.

${ }^{7}$ Los grafos que forman parte de este artículo han sido realizados con el software Gephi (versión 0.8.2).

${ }^{8}$ Reynoso, Carlos. Redes sociales y complejidad: modelos interdisciplinarios en la gestión sostenible de la sociedad y la cultura (Buenos Aires: Editorial SB, 2011), p. 60. 
Los mapas y los grafos no se conciben aquí como ilustraciones del texto, sino que son parte de la estrategia metodológica en el proceso de formulación y de revisión de las hipótesis propuestas ${ }^{9}$. Por tanto, nuestro objetivo es analizar el modo en que se tejieron las relaciones de solidaridad y de alianza que configuraron los grupos sociales; en este caso, interesa ver a los sujetos dentro de estas relaciones de interdependencia, las cuales condicionaron sus acciones e influyeron necesariamente sobre sus objetivos. La estructura de relaciones no solo fue dinámica sino que además condicionó los itinerarios sociales y hasta las oportunidades o las posibilidades de éxito ${ }^{10}$.

Por otra parte, consideramos que el concepto de red social presenta una serie de ventajas en relación a otras perspectivas, en tanto que la interpretación histórica a partir de redes busca acercarse a un tipo de comprensión que evite las excesivas generalizaciones o la partición de los grupos sociales mediante categorías agregativas que licuan las especificidades y en la que se trate de soslayar el forzamiento en la clasificación y la descripción de prácticas sociales a partir de esquemas explicativos previos.

La metodología de ARS nos permitió reconstruir parcialmente a las redes de solidaridad étnicas e indagar sobre la construcción de las zonas de influencia de los bolicheros sirios y libaneses, avanzando en la reconstrucción de una cartografía de sus redes familiares y de negocios ${ }^{11}$. De esta manera, identificamos el funcionamiento de solidaridades especiales dentro del grupo que favorecieron sus negocios y el funcionamiento de consensos que evitaron la superposición territorial o comercial aunque ofrecieran un mismo servicio. Los grafos de redes reflejan la capacidad dinámica que tuvieron los sirios y libaneses para moverse, intentando acceder y disputar recursos escasos, como primer eslabón en la construcción de una territorialidad bolichera y, entendiendo a la territorialidad desde una concepción relacional del espacio previo al territorio, definida por Robert Sack, como la "[...] estrategia de un individuo o grupo de afectar, influir o controlar personas, fenómenos y sus relaciones, a través de la delimitación y ejerciendo

\footnotetext{
${ }^{9}$ Vezub, Julio. Valentín Saygüeque y la gobernación Indígena de las Manzanas, Poder y etnicidad en la Patagonia septentrional (1860-1881) (Buenos Aires: Prometeo, 2009), p. 65.

${ }^{10}$ Para el caso de Neuquén, Favaro e Iuorno han analizado en una clave similar, cómo las relaciones de parentesco y las redes familiares permitieron que los inmigrantes libaneses y sirios realizaran una acumulación mercantil durante las primeras décadas del siglo XX, legitimando luego su inserción política a partir de la participación activa en asociaciones intermedias y finalmente, consolidándose como parte de los sectores social y políticamente dominantes de la provincia de Neuquén a partir de la segunda mitad de siglo, con el objetivo de precisar la diversidad al interior de estos grupos étnicos y destacar las diferencias regionales dentro de la provincia. Favaro, Orietta e Iuorno, Graciela. "Libaneses y sirios. Actividades comercial y participación en el espacio público", en Entrepasados, n. ${ }^{\circ}$ 17, Buenos Aires, 1999, pp. 27-47. Favaro, Orietta e Iuorno, Graciela. "Entre territorio y provincia. Libaneses y sirios, comercio y política en Neuquén”, en Favaro, Orietta (ed.), Neuquén, la construcción de un orden estatal, (Neuquén: Centro de Estudios Históricos de Estado, Política y Cultura, 1999).

${ }^{11}$ Miceli y Guerrero han recurrido al ARS para reconstruir genealogías en la comunidad de El Chalía y destacan "[...] la enorme capacidad de gestación de hipótesis y de posterior control de estas que la aplicación de estas perspectivas comporta". Miceli, Jorge y Guerrero, Sergio. "Redes libres de escala y su uso en el análisis de datos etnográficos: el caso de la comunidad tehuelche del Chalía", en Díaz, Diego, et al. (comps.), Exploraciones en antropología y complejidad (Buenos Aires: Editorial SB, 2007), p. 190.
} 
control sobre una geografía"12. Este tipo de territorialidades son configuraciones que pueden adoptar forma de zonas, de redes o de lugares y que pueden devenir territorios, a partir de los cuales se transforman en áreas delimitadas, cercadas o en disputa.

Imagen 1. Relaciones interpersonales y entre personas y parajes (1911-1940).

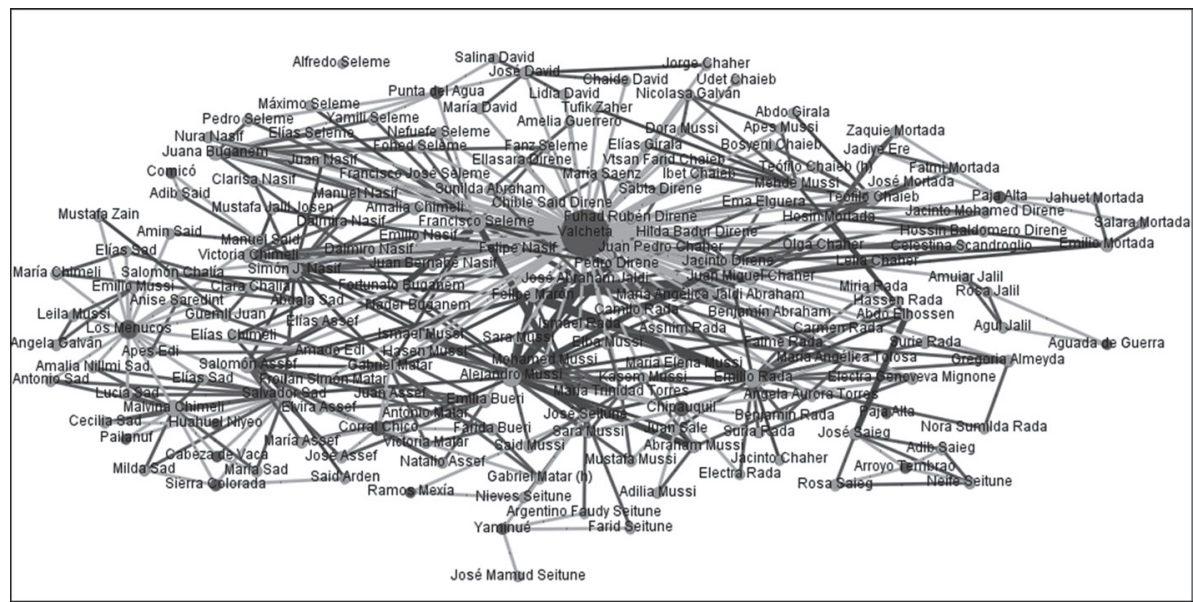

Fuente: elaboración de los autores a partir de datos relevados en la delegación del Registro Civil de la localidad de Valcheta.

En este primer grafo se muestran las relaciones entre personas y parajes que surgieron de los datos relevados en el Registro Civil de la localidad de Valcheta, correspondientes a las actas de matrimonio y las partidas de nacimiento allí asentadas entre 1911 y 1940.

En un tono más oscuro se observan los nodos que representan las localidades, mientras que en celeste aparecen aquellos que representan personas.

Establecimos las articulaciones entre los nodos de acuerdo a los siguientes criterios:

1. Territorialidad (líneas más claras): se establecieron vínculos entre personas y lugares solamente cuando el documento explicita esa relación. No se extrapola la residencia atribuida a un sujeto a sus familiares si este dato no aparece en los documentos relevados.

2. Vínculos entre personas (líneas oscuras):

a) Parentesco: las conexiones entre personas indican relaciones de filiación y matrimoniales. Para no sobrecargar el grafo, otros vínculos de parentesco pueden seguirse indirectamente.

\footnotetext{
${ }^{12}$ Citado por Benedetti, Alejandro. "Territorio: concepto integrador de la geografía contemporánea", en Souto, Patricia (coord.), Territorio, lugar, paisaje. Prácticas y conceptos básicos en geografia (Buenos Aires: Editorial de la Facultad de Filosofía y letras de la Universidad de Buenos Aires, 2011), p. 45.
} 
Redes sociales y territorialidad bolichera de sirios y libaneses en el sudeste de Río Negro,...

b) Familiarización ${ }^{13}:$ se establecen vínculos entre las personas que aparecen como testigos y/o apoderados en las actas de nacimientos y matrimonios y aquellos varones ${ }^{14}$ que aparecen como padres o esposos.

El tamaño de los nodos varía de acuerdo a la cantidad de relaciones que articulan (siempre en función del relevamiento documental realizado). Así, los nodos de localidades o parajes más grandes son aquellos asociados a la mayor cantidad de personas, mientras que los nodos de personas de menor tamaño son aquellos que se relacionan con menores redes de parientes o que articulan redes que incluyen diferentes parajes.

Asimismo, el análisis de diferentes sectores de este grafo general permite una aproximación a las redes de parientes en las que se insertaban diferentes sujetos con el propósito de responder las cuestiones sobre ¿qué redes se conectaban entre sí?, ¿coexistían en torno a los mismos espacios geográficos?, etc. O también puede permitirnos ver las estrategias de dispersión espacial o la movilidad a lo largo del tiempo de algunas redes de parientes, como en el caso del matrimonio entre Salvador Sad y Malvina Chimeli y los diferentes lugares en donde se los registra en su casamiento y los nacimientos de sus hijos.

Una de las maneras de identificar a las familias más respetadas y con mejor posición económica surgió de la consulta de la nómina de miembros directivos del Centro Libanés-Argentino de Valcheta en 1958, teniendo en cuenta la elección de los sujetos que se consideraban más representativos de la colectividad.

\author{
Presidente: Felipe Marón \\ Vice-presidente: José H. Mortada \\ Tesorero: José Yeber \\ Protesorero: Abdo Girala \\ Secretario: Ali Chible \\ Prosecretario: Mehde Mussi
}

Todos los nombrados pertenecían a familias tradicionales en la región, prosperaron y llegaron a ocupar lugares destacados en la comunidad. A los anteriores, habría que sumar a Juan Miguel Chaher, presidente de la comisión municipal de Valcheta entre 1933 y 1937. Chaher se casó con Celestina Scandroglio, hija de Carlos Scandroglio vecino notable y una de las personas más influyentes de la zona a comienzos del

\footnotetext{
13 Tomamos la idea de familiarización a partir de Ramos, Ana. Los pliegues del linaje: memorias y politicas mapuches- tehuelches en contextos de desplazamientos (Buenos Aires: Eudeba, 2010), pp. 29-31. En nuestro caso, no consideramos que estos lazos impliquen una incorporación al linaje en los mismos términos que la filiación, pero sí que dan cuenta de la confirmación de una alianza en la realización de estos actos. Así, varios de los que aparecen como testigos de los nacimientos son socios comerciales de los padres del recién nacido. Por lo tanto, proponemos un uso parcial del concepto de familiarización, entendiendo que resulta operativo para el análisis de redes no mapuches.

${ }^{14}$ Tanto en las actas de Registro Civil como en la bibliografía y los relatos familiares se evidencia que la continuidad del linaje se traza a través de la línea patrilineal. Por otra parte, el espacio de lo público es en la región y en el momento histórico estudiado desde un ámbito eminentemente masculino.
} 
siglo XX. Para 1904 Scandroglio, comerciante de origen italiano ${ }^{15}$, estaba radicado en Valcheta como comerciante, con campo, 40 bueyes, 70 vacas, 24 caballos, 2 carros y 1.000 ovejas, lo que lo convertía en una de las personas más acaudaladas de la zona.

Además, Juan Jacinto Scandroglio, cuñado de Juan Miguel Chaher, concentró las actividades estatales más importantes durante el período en que era juez de paz ${ }^{16}$, encargado del registro civil y estaba bajo su jurisdicción todos los asuntos civiles hasta determinada suma. Además, los jueces de paz:

[...] tenían también mucho control sobre el ámbito de la producción y el comercio. Eran los que extendían las guías de campaña que se utilizaban para transportar ganado y frutos del país dentro y fuera del Territorio, debían registrar las marcas del ganado que definían su propiedad y también cobrar las patentes a los comercios de la localidad. Mensualmente estos funcionarios debían remitir a la Gobernación un balance haciendo constar los datos de todo el movimiento que había habido en la oficina ${ }^{17}$.

La amplia influencia de los jueces de paz fue señalada por el inspector de tierras que realizó el informe sobre la zona de Valcheta en 1920 y quien enfatizaba la autoridad de los jueces de paz y su ascendencia sobre los vecinos, en contraste con la actitud crítica que se tenían hacia la policía:

Es de mencionar el poco respeto que a la policía se le tiene, viendo en ella los habitantes a un enemigo en vez de considerarlos como guardianes del orden público encargado de velar por sus vidas y haciendas. Contrasta esto con el profundo respeto -mezcla de temor- que se tiene al Juez de Paz ${ }^{18}$.

Posiblemente, Juan M. Chaher se benefició del poder y de la influencia de los Scandroglio en la región. Pero habían otros sirios y libaneses destacados, uno muy importante fue Nader Buganem, para reflejar su status social recurrimos a la misma fuente: el inspector de tierras era muy crítico a la hora de valorar las viviendas de la región, aunque valoró positivamente a la propiedad de Buganem:

\footnotetext{
${ }^{15}$ Debemos señalar que este tipo de relaciones exógenas no fueron excepcionales para los sirio-libaneses, aunque el análisis de ese tipo de articulaciones no sea central en nuestro estudio.

${ }^{16}$ Graciela Suarez describe el importante poder de los jueces de paz en el período de los Territorios Nacionales en Río Negro, acentuando la falta de control del poder central sobre el ejercicio de justicia. Suárez, Graciela. "La Justicia Letrada: su organización y funcionamiento en el Territorio Nacional de Río Negro, 1879-1915”, en Ruffini, Martha y Masera, Ricardo (coords.), Horizontes en perspectiva. Contribuciones para la Historia de Río Negro 1884-1955 (Viedma: Fundación Ameghino, 2007), pp. 247-270.

${ }^{17}$ Debattista, Susana y Destéffaniz, Carolina. "El difícil arte de juzgar y ser justo", en Gentile, María Beatriz; Rafart, Carlos Gabriel y Bohoslavsky, Ernesto (comp.), Historias de sangre, locura y amor, Neuquén (1900-1950) (General Roca: Publifadecs, 2000), p. 207. Además, las autoras analizan casos que demuestran la convivencia de intereses entre jueces de paz, policías y bolicheros en Neuquén, durante la primera mitad del siglo XX.

${ }^{18}$ AHPRN, Sección Inspección de la Dirección General de Tierras en las zonas de influencia I y II de Valcheta, 1920, p. 20.
} 
Redes sociales y territorialidad bolichera de sirios y libaneses en el sudeste de Río Negro,...

Son en su mayoría construcciones chatas y sin estética, de una altura no mayor de tres metros y casi siempre compuestas de una pieza y cocina.

Las únicas construcciones dignas de mención son las del Señor Salvador Sedda, Club Social, Nader Buganem y J. Casas en Valcheta y lo de la Señora Ángela Ruiz de Luque que ha construido un confortable chalet en Corral Chico.

En general no se ha hecho -respecto a construcciones- más que cumplir con le [sic] ley de concesión que exije [sic] una pieza y cocina de material y después se han ido aumentando piezas de acuerdo a las necesidades de las familias, sin tener para nada en cuenta un plan de edificación ${ }^{19}$.

Otra evidencia de la valoración positiva de Buganem se describe en un documento periodístico del 10 de agosto de 1939 expuesto en el Archivo del Museo Regional María Inés Koop de la Ciudad Valcheta. En el periódico se transcribe un discurso pronunciado por Nader Buganem en representación de la colectividad libanesa ante el gobernador de Río Negro Adalberto Pagano, en el que agradeció las gestiones realizadas en favor de la comunidad y en la cual solicitó obras de riego que favorecieran a los agricultores. Seguramente parte del renombre de Buganem radicó en su calidad de agricultor; la actividad mercantil y la ganadería tuvieron siempre una valoración negativa para los agentes estatales.

Otro ejemplo de un vecino libanés influyente fue el de Alejandro Mussi (originalmente Apes Musa Arba), quien fue un próspero comerciante que se destacó por sus vinculaciones políticas con el consulado libanés en Buenos Aires. Dichos contactos habrían favorecido la inserción comercial de muchos inmigrantes del Cercano Oriente, mediante el adelanto de mercadería para que vendieran como mercachifles en la campaña, lo que generó lazos de reciprocidad y lo dotó de una notable influencia.

Pese a los casos mencionados de mayor éxito e influencia, y aunque no se trató de un colectivo inmigratorio homogéneo, la mayoría de los inmigrantes sirios y libaneses pertenecía a los sectores subalternos. La gran mayoría de los bolicheros sirios y libaneses funcionaron como intermediarios comerciales, operando con eficacia en una geografía compleja y tendieron redes que se superpusieron con las antiguas rutas indígenas y se ubicaron en los espacios periféricos para las grandes casas comerciales.

Aunque es muy difícil obtener pruebas documentales concluyentes que indiquen el reparto territorial, podemos sostener, a partir de la aplicación de la metodología de ARS y los grafos resultantes, que existió una distribución del territorio y de los mercados entre los sirios y libaneses, y luego entre sus descendientes, sobre la base de acuerdos que eran mayormente tácitos. Las evidencias ratifican el funcionamiento de solidaridades especiales dentro del grupo étnico y niegan conflictos importantes por la ocupación del espacio, a la hora de favorecer los negocios de compatriotas con adelantos de mercadería, préstamos especiales, favores en trámites y paliativos en épocas de crisis.

\footnotetext{
${ }^{19}$ AHPRN, Sección Inspección de la Dirección General de Tierras en las zonas de influencia I y II de Valcheta, 1920, p. 18.
} 
Un ejemplo concreto de los favores especiales se evidencia en los varios casos relevados, donde los inmigrantes sirios o los libaneses anotaron al hijo de un amigo o socio mediante una carta poder para evitar el viaje hasta el Registro Civil de Valcheta. Si bien se suponía el funcionamiento de un registro móvil en la campaña, en la práctica el servicio no funcionó y la denuncia de los nacimientos se demoraba. Por ejemplo, Gabriel Matar y Francisco Seleme de la zona de Corral anotaron hijos de Salomón Assef y Mustafa Zain de Los Menucos anotó hijos de Salomón Chalía. Este tipo de favores seguramente funcionaban en el marco de reciprocidades más amplias.

Imagen 2. Relaciones de familiarización/alianza (1911-1940).

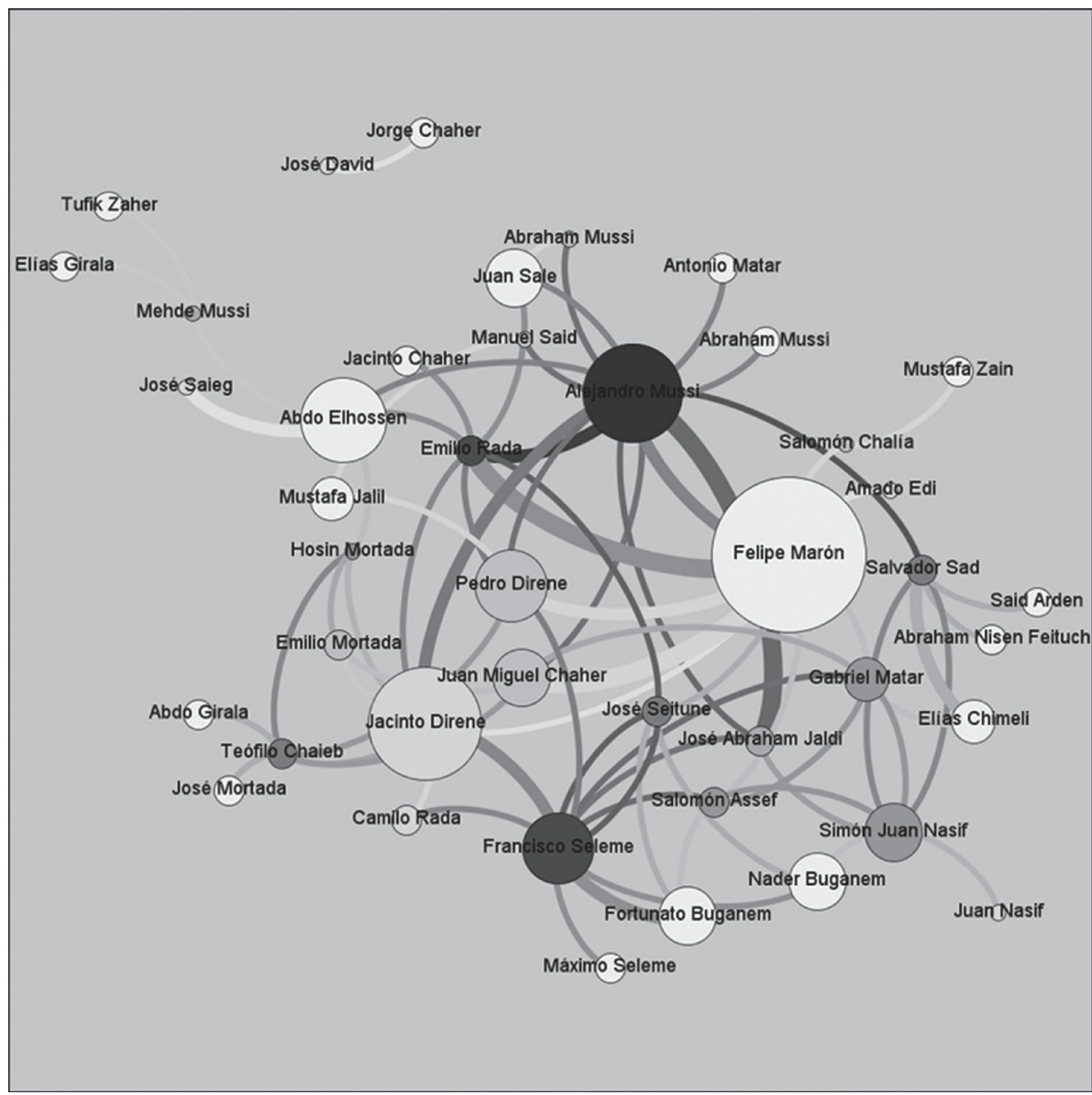

Fuente: elaboración de los autores a partir de datos relevados en la delegación del Registro Civil de la localidad de Valcheta.

Este segundo grafo muestra solamente las relaciones de familiarización/alianza que aparecían en el anterior. El tamaño de los nodos será mayor en función de la cantidad de veces en las que esa persona actuó como testigo o como apoderado de otro, mientras que la intensidad del color crece en función de la mayor cantidad de eventos 
en los que un tercero ofició como testigo de la paternidad o el matrimonio de una persona. Por último, el grosor y la intensidad de color de las aristas varían en función de la cantidad de veces que se vincularon los sujetos conectados a través de este tipo de eventos, buscando mostrar aquellos casos en que la alianza es reconfirmada en sucesivas ocasiones. Vemos entonces cómo se destacan en el registro documental figuras como Felipe Marón (quien apareció como testigo en más ocasiones que ningún otro), Alejandro Mussi (quien convocó a más testigos, y asimismo, actuó como testigo en varios eventos) y otros apellidos como Chaher, Direne o Buganem. Sin inferir ningún mecanicismo a partir de ello, cabe señalar cierta tendencia a que coincidan los personajes buscados como testigos con aquellos que otras fuentes señalan como exitosos social y económicamente.

Al retomar las redes familiares de sirios y libaneses en la zona de Valcheta, es interesante la movilidad comercial de los hijos, que muy pocas veces superpusieron sus negocios con el de los padres: en sus propios términos, prefirieron ocupar espacios mercantiles vacantes. Por ejemplo, Antonio Sad tuvo su boliche en Sierra Colorada y su hijo Salvador Sad decidió trabajar comercialmente en Los Menucos y Cabeza de Vaca. Otro caso es el de Adib Saieg, hijo de José Saieg, mientras su padre tuvo boliche en Arroyo Tembrao, Adib se mudó hasta Aguada Cecilio para establecerse. Un último ejemplo fue el de Mustafa Mussi, quien decidió operar comercialmente en Treneta, mientras su padre Mehde Mussi se mantuvo en Paja Alta.

\section{Redes sociales y territorialidad bolichera}

Los bolicheros sirios y libaneses tuvieron particularidades en su modo de operar que nos permiten discutir los estereotipos más comunes asociados a sus actividades económicas $^{20}$. Además, nos interesa reflexionar sobre la relación establecida por los comerciantes sirios y libaneses con los agentes del estado. Estos funcionarios contribuyeron a establecer la supuesta sinonimia en el sentido común patagónico entre boliches sirios y libaneses:

Los pobladores existentes en las zonas I y II de Influencia y Pueblo y Colonia Valcheta pueden dividirse en dos grandes grupos: cristianos e indígenas. Entre los primeros están representadas distintas nacionalidades: argentinos, turcos. Italianos, españoles, chilenos, etc. [...]

Los cristianos se encuentran en su mayoría en el pueblo y Colonia Valcheta y se dedican unos a la agricultura, otros al comercio y muy pocos a la ganadería. En cambio los indígenas constituyen la inmensa mayoría de los pobladores de los lotes pastoriles y se dedican pura y exclusivamente a la ganadería. Uno que otro turco se encuentra en los lotes pastoriles, dedicado al comercio ${ }^{21}$.

El comentario del agente de estado es muy sugerente respecto al supuesto lugar ocupado por los sirios y por los libaneses en la clasificación de las ocupaciones por

\footnotetext{
${ }^{20}$ Autor, 2013.

${ }^{21}$ AHPRN, Sección Inspección de la Dirección General de Tierras en las zonas de influencia I y II de Valcheta, 1920, pp. 15-16. Subrayado en el original.
} 
grupo étnico ${ }^{22}$. En buena medida, se continuaba reproduciendo la estigmatización forjada por Juan Alsina ${ }^{23}$ sobre el "turco vendedor de baratijas". No obstante, el inspector refleja una particularidad del comercio de los sirios y los libaneses: la de operar en medio de los lotes pastoriles con fines estrictamente comerciales.

En la clasificación de pobladores, los sirios y los libaneses aparecían como un sujeto híbrido, que generaba una contradicción a la dicotomía habitualmente planteada entre agricultores cristianos y ganaderos indígenas ${ }^{24}$. Los bolicheros sirios y libaneses se dedicaban al comercio, una actividad de valoración intermedia para los agentes estatales. Los inmigrantes turcos no eran para los inspectores tan negativos como los pobladores indígenas, aunque tampoco deseables como los extranjeros de origen europeo. La construcción dicotómica entre cristianos e indígenas se reforzaba con la dualidad entre la ciudad y el campo: si en el ámbito rural predominaban los crianceros ${ }^{25}$ de origen indígena, en los cascos urbanos eran, en su mayoría, los cristianos de origen diversos.

En la Colonia y Pueblo Valcheta se encuentra la población que podríamos llamar civilizada, predominando entre ellos los Turcos que se dedican de preferencia al comercio que en esas zonas produce pingües beneficios. El italiano y el español, dedicados a la agricultura están en minoría y sus costumbres y modalidades por todos conocidas, hacen de estos elementos un factor de progreso cuya radicación en las zonas, interesa al gobierno fomentar ${ }^{26}$.

La distinción entre turcos y europeos era evidente, dado por sobreentendido el valor superior de las costumbres y modalidades de los italianos y españoles, grupos migratorios que había que estimular (por oposición a los sirios y a los libaneses, había que desalentarlos). Además, el documento suma otra especificidad a los comerciantes sirios y libaneses, ya que no solo operaban entre crianceros, sino que lo hacían por importantes márgenes de ganancia. Las dificultades de trabajar entre crianceros humildes en un territorio difícil de transitar por su geografía, pueden ayudarnos a explicar la opción de los mercachifles y los bolicheros sirios y libaneses, y las abstenciones de explotar ese mercado por parte de las grandes casas comerciales de la zona.

\footnotetext{
${ }^{22}$ La clasificación de sirios y libaneses como turcos surgió a partir de su procedencia de territorios dominados por el Imperio Otomano en los primeros años del siglo XX, lo que determinó que dichos inmigrantes arribaran al país con pasaportes bajo esa denominación; la identificación como turcos de grupos étnicos árabes se reprodujo erróneamente en documentos censales y se popularizó con cierta carga peyorativa en el interior patagónico.

${ }^{23}$ Alsina, Juan. La inmigración en el primer siglo de la independencia (Buenos Aires: editado por Felipe S. Alsina, 1910), p. 91.

${ }^{24}$ Autor, 2014.

${ }^{25}$ Cuando hablamos de crianceros en la región patagónica, nos referimos a pequeños productores ganaderos de ovinos o de caprinos en pequeña escala, generalmente en el marco de una economía familiar.

${ }^{26}$ AHPRN, Sección Inspección de la Dirección General de Tierras en las zonas de influencia I y II de Valcheta, 1920, p. 17.
} 
Si los inspectores de tierras veían con pesimismo los emprendimientos comerciales en general, peor era su visión sobre los boliches, los que eran equiparados en sus informes con las pulperías de la campaña bonaerense, asociadas directamente a la violencia y a los conflictos. De esta manera, los bolicheros eran considerados comerciantes embaucadores que profundizaban el atraso de la población local y que alentaban el juego y los vicios. Ya desde 1904 aparecen amonestaciones al comercio bolichero, sin precisar el origen étnico de sus propietarios, aunque señalando su carácter de extranjeros.

Uno de los lujos de estos pobladores son los parejeros; no hay choza á donde uno llegue que no se encuentre un parejero atado á las estacas y con ellos concurren á las pulperías los domingos ó días de reunión a entregarse á su goce predilecto en el cual son fomentados por los Pulperos, verdaderos culpables por ser extranjeros en su totalidad y más civilizados, pero que ávidos de riquezas y poco escrupulosos no desdeñan explotar este filón para ir haciéndose poco á poco dueños de todos sus bienes. Excuso decir que estas reuniones terminan con sendas jugadas al monte, choclón, y demás juegos prohibidos de azar, lo más común es ver allí una carrera por 500 vacas ó igual número de ovejas; esta es la realidad exacta y tristísima del carácter de los pobladores de esa Colonia $[\ldots]^{27}$.

En este pasaje del documento se cristalizan muchos de los estereotipos negativos sobre los bolicheros: comerciantes timadores que se aprovechan del supuesto atraso de los pobladores y que por su carácter civilizado, eran los verdaderos responsables de los vicios. A su vez se denuncia el objetivo de la lisa y llana expropiación progresiva de los bienes de los crianceros que perdían su capital en los juegos asociados al boliche. Es interesante observar cómo se reduce en este informe el funcionamiento del boliche a la de un bar o fonda, en donde se relegan las funciones de almacén.

Para 1920 eran muchos los comerciantes sirios y libaneses que operaban en el sudeste de Río Negro, algunos como bolicheros establecidos en las principales ciudades (Valcheta, San Antonio, Sierra Grande), otros como bolicheros o mercachifles en los muchos parajes en las cercanías de esas ciudades. De los datos relevados surge que la mayor parte de estos emprendimientos eran casas comerciales medianas o pequeñas, excepto una: Chaher y Cía., casa comercial radicada en San Antonio y con representantes comerciales en Valcheta. De acuerdo al inspector de tierras interviniente, "[e]l comercio existente en el pueblo está representado por fuertes firmas tales como las de Podestá y Cia, Sociedad Anónima Exportadora e importadora de la Patagonia, Chaher y Cia, Salvador Sedda, etc etc. La mayoría de estas casas con sede central en Buenos Aires y San Antonio y dedicadas todas ellas a ramos generales" ${ }^{28}$. Esas grandes casas comerciales controlaban buena parte del mercado de la zona, aunque contaban con la desventaja relativa de esperar que los clientes se acercaran a comprar a las principales ciudades. En contraste, los comerciantes sirios y libaneses -y también sujetos pertenecientes a otros colectivos migratorios- operaban

\footnotetext{
${ }^{27}$ AHPRN, Sección Inspección de la Dirección General de Tierras en las zonas de influencia I y II de Valcheta, 1904, pp. 9-10.

${ }^{28}$ AHPRN, Sección Inspección de la Dirección General de Tierras en las zonas de influencia I y II de Valcheta, 1920, p. 5.
} 
mayoritariamente, acercándose al lugar de residencia de los potenciales clientes, hasta asentarse en los parajes donde existía un mercado vacante.

Un ejemplo en este sentido, es el de José Seitune, libanés que se radicó en primera instancia en San Antonio, desde donde comenzó a vender como mercachifle por la línea sur de Río Negro hasta establecerse definitivamente con boliche en la zona de Yaminué, cuando consideró que había un conjunto de pobladores pasibles de convertirse en clientes permanentes.

De los informes de los inspectores de tierras surge que todos los productos y los frutos del país producidos por los crianceros del sudeste de la provincia, eran acopiados (con o sin intermediarios) por casas de comercio en San Antonio. El transporte se hacía en carretas de bueyes y carros tirados por mulas.

Por su parte, los inspectores denunciaban que los precios pagados a los pobladores por sus productos eran excesivamente bajos, aun considerando la supuesta baja calidad de los mismos: el precio de los frutos se pagaba a un tercio de su valor real. Los comerciantes se justificaban, alegando que necesitaban reducir los gastos de los fletes hasta San Antonio, el costo de la carga en los vapores y su depósito en destino, es decir en Buenos Aires.

Debemos señalar que no se detectaron aún conflictos importantes en la competencia entre los comerciantes sirios y libaneses y las grandes casas comerciales en la competencia por un mercado común. Al parecer, los comerciantes sirios y libaneses funcionaron como intermediarios comerciales y llegaron hasta donde las grandes casas comerciales no lo hacían.

Una virtud concreta de los comerciantes sirios y libaneses fue su capacidad de operar con eficiencia en una geografía compleja. Las fuentes son elocuentes sobre las dificultades del territorio: terrenos inestables, mesetas quebradas y muy pedregosas, difíciles de recorrer, sumado a las muchas leguas de territorios sin aguadas entre parajes. Dichos parajes sobre los que se establecieron los comerciantes sirios y libaneses tendieron a rodear la meseta del Somuncurá, formando un contorno mercantil en los márgenes de la formación mesetaria.

La inserción comercial de los sirios y los libaneses se produjo, prácticamente, en todos los casos, a partir de lazos familiares. Por ejemplo, de la familia Mussi llegaron cinco integrantes desde Líbano, los hermanos Abraham, Ale y José, el primo de ellos, Alejandro, y un hijo de este último, Mehde. Pero el arribo no fue simultáneo sino que se produjo en cadena migratoria. El recién llegado solía ingresar como dependiente comercial de un familiar o funcionaba como revendedor en beneficio de este por unos años, hasta formar un capital propio e independizarse, instancia en la que el ciclo podía recomenzar con un nuevo inmigrante.

Otro caso en el cual los lazos familiares se imbricaron con los intereses comerciales fue el de Julián Luna, inmigrante libanés que inicialmente se radicó en Chile, donde 
contrajo matrimonio con Inés Helguera. A través de la promoción de la hermana de Inés -Ema- y de un amigo de Líbano -Jacinto Mohamed Direne-, ambos radicados en Valcheta, se decidió migrar a Río Negro. El hermano de Jacinto, Mislev Direne, comerciante local, favoreció los préstamos y los adelantos de mercadería necesarios para que pudiese realizar intercambios entre las zonas de Nahuel Niyeu y en El Salado.

En cuanto a la competencia al interior del grupo étnico, los testimonios apuntan a que los bolicheros sirios y libaneses trataban de no superponerse aunque ofrecieran un servicio similar. Por ejemplo, en Valcheta funcionaron simultáneamente tres hoteles que eran propiedades de sirios y libaneses, aunque ofrecían diferentes tarifas y comodidades. El Hotel Marón, de Felipe Marón, era el considerado de mejor calidad y el que tenía las tarifas más elevadas. Luego el Hotel Rada, de Emilio Rada (luego de Ismael Rada), que era un hotel más modesto y por último el bar-hotel de Jalil Dana, que era el más económico. El caso de los hoteles demuestra la diversidad de servicios ofrecidos por los comerciantes sirios y libaneses, controlando en ese caso el mercado hotelero en sus diferentes segmentos.

Aunque hayamos identificado a los bolicheros sirios y libaneses en una localidad o en un paraje en concreto, vemos que su ubicación pudo variar a lo largo de los años. En el siguiente grafo hemos distribuido los diferentes parajes mencionados en los documentos en sus ubicaciones geográficas relativas, con el objetivo de mostrar las movilidades entre parajes de algunos sujetos, pero también casos como el de Emilio Rada, en el que su vinculación con dos parajes diferentes fue simultánea, dando cuenta no de un desplazamiento sino de una movilidad que fue parte de la conformación de su red de relaciones.

Imagen 3. Movilidad de algunos bolicheros dentro de la región estudiada.

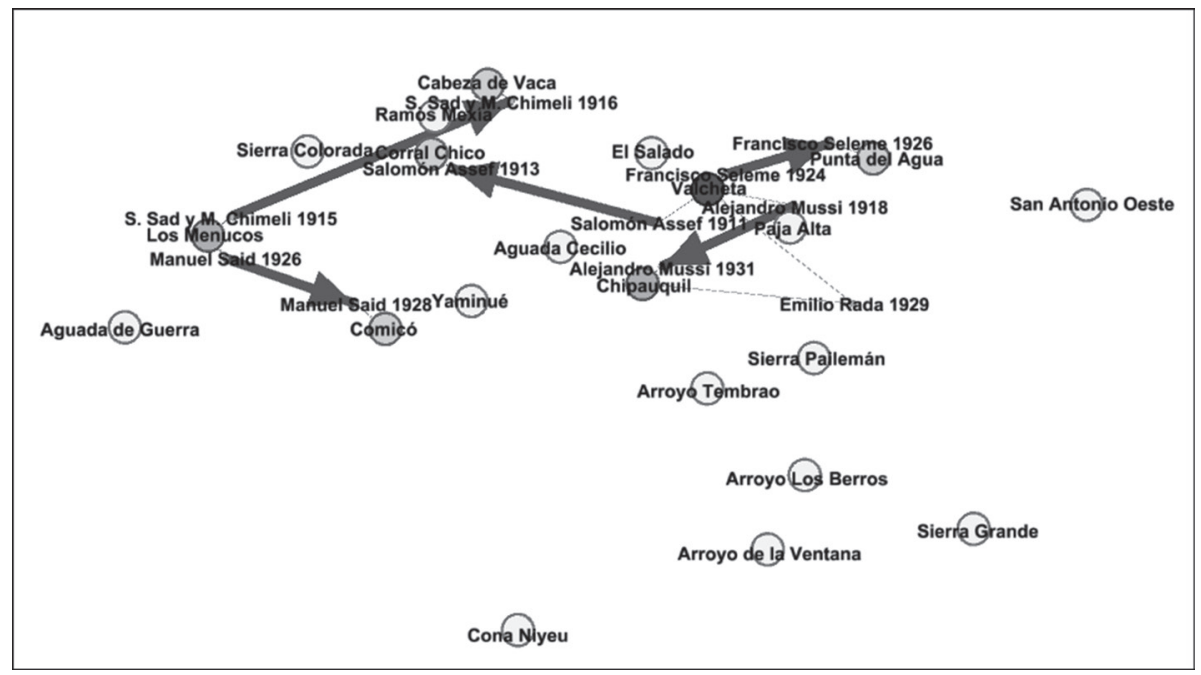

Fuente: elaboración de los autores a partir de datos relevados en la delegación del Registro Civil de la localidad de Valcheta, inspecciones de la Dirección General de Tierras y Registro Único de Comercios (AHPRN). 
Una de las constantes identificadas es que en muchos casos los bolicheros abandonaron los parajes más alejados para garantizar la educación de sus hijos. Aun cuando económicamente era menos conveniente, decidieron mudarse a Valcheta o lugares más cercanos a las localidades más importantes. Un caso concreto es el de Emilio Rada, quien formó su capital en la zona de Chipauquil hasta mediados de la década del veinte, para luego establecerse en Valcheta y hacer estudiar a su hijos (Electra, Ismael, Miria, Hasen, Suría, Alba, Fatúm, Orlando y Mirta). En dicha localidad instaló su Boliche Rada que era una combinación de carnicería, almacén, hotel y bar. Vale aclarar que Rada mantuvo, no obstante, sus intereses ganaderos en la zona rural, donde trabajó 600 hectáreas.

Otro ejemplo es el de Mustafa Mussi, quien continuó con el negocio de su padre (Mehde) en Treneta hasta que sus hijos tuvieron la necesidad de asistir al colegio. Entonces se asentó en Valcheta y estableció un boliche en el pueblo. En este caso, los intereses mercantiles en el paraje de origen se abandonaron.

Un último caso - diferente- es el de Jadiye Ere, quien permaneció con sus hijos en Valcheta atendiendo una pequeña tienda, mientras su marido mantuvo el boliche en Paja Alta, lo que hizo posible que sostuvieran los intereses comerciales en los dos espacios.

\section{La rearticulación de las redes y los posibles límites de la decadencia bolichera de sirios y de libaneses}

Por último, nos interesa aproximarnos a la discusión sobre la pervivencia o la reconversión de las redes sociales y comerciales de sirios y de libaneses luego de 1940, a partir de la revisión de las perspectivas sobre la problemática centradas en el aspecto económico. Para ello mantendremos el acento en las redes familiares, sin avanzar en una reconstrucción económica más allá de los datos suministrados por los registros censales de comercios.

En definitiva, nuestras observaciones buscan favorecer una revisión de los enfoques dominantes sobre la última etapa de los boliches en el sur de Río Negro y el norte de Chubut. Distintos estudios ${ }^{29}$ sobre los boliches en la región patagónica señalaron a las décadas de 1930 y 1940 como la coyuntura, a partir de la cual este tipo de comercios habrían entrado en decadencia para luego extinguirse progresivamente. Desde esa línea argumental, la declinación de los boliches se habría producido a partir de los efectos devastadores de la crisis de 1929, sumados al perjudicial desarrollo del transporte automotor (que hacía obsoleto el paso por los boliches), a los efectos nocivos de la aparición de nuevas rutas que reemplazaron a las antiguas huellas y picadas, y por último, al aumento de la monetización, los cual reducía los márgenes de beneficios que se obtenían mediante el trueque. Aunque estas hipótesis son discutibles, incluso en el plano estrictamente económico (teniendo en cuenta que la monetización existía

\footnotetext{
${ }^{29}$ Un ejemplo posible es el trabajo de Ibarra, Horacio y Hernández, Carlos. "Huellas, picadas, carros, boliches. Poblamiento y comunicaciones en la Patagonia vieja", en VI Congreso de Historia Social y Politica de la Patagonia argentino-chilena, Trevelin, 2007.
} 
Redes sociales y territorialidad bolichera de sirios y libaneses en el sudeste de Río Negro,...

en el primer cuarto del siglo XX, además de que el trueque se mantuvo luego del período), entendemos que no se trató de un proceso tan lineal y unívoco.

Consideramos que el caso del sudeste de Río Negro permite matizar la hipótesis con mayor acento en lo económico, ya que si bien es cierto que tiende a reducirse el número de boliches en la zona rural - probablemente a partir del proceso de migraciones del campo a las ciudades ${ }^{30}$ - los boliches no desaparecen completamente. Al seguir la lógica de la urbanización, se puede inferir que estos tienden a concentrarse en las ciudades pero mantienen sus clientes en las zonas rurales.

El cuadro 1 refleja la pervivencia de los comercios de sirios y de libaneses en la zona. De los treinta comercios registrados en la ciudad de Valcheta para los años 1953-1955, diez eran propiedad de sirios y de libaneses o de sus descendientes directos, es decir que mantenían el control de un tercio de la participación mercantil total.

El número de comercios de propietarios sirios o libaneses puede ampliarse si sumamos los de la zona de influencia de Valcheta, donde para la década de 1950 figuraban cuatro comercios de bolicheros en parajes cercanos asentados en el Registro Único de Comercios de la Provincia de Río Negro, específicamente nos referimos a los negocios minoristas de Abdo El Hossen (Sierra Pailemán), Adib Saieg (Aguada Cecilio), Derecho Mussi (Aguada Cecilio) y Antonio Sad (Sierra Colorada).

Probablemente, los argumentos económicos funcionaron de forma efectiva y los boliches se vieron afectados por las consecuencias de la crisis mundial de 1929. Asimismo, es posible que hayan reducido su margen de beneficio a partir de la mayor monetización de la economía (que reducía las ganancias realizadas a partir del trueque) y seguramente debieron adaptar sus estrategias al proceso de urbanización de la zona. No obstante, incluso basándonos en fuentes que no registraron la totalidad de los comercios existentes, parecen haber sufrido una reconversión más que una desaparición absoluta.

Sin haber avanzado en la reconstrucción estrictamente económica, es claro que los boliches -en tanto comercios rurales-fueron disminuyendo en su número, hasta casi desaparecer de la región. Pero esto no quiere decir que esos negocios y sus dueños hayan abandonado la actividad mercantil sino que re-direccionaron sus intereses hacia las localidades más pobladas de la línea sur. Esa transformación de la lógica mercantil, necesariamente debe haber afectado la conformación y la orientación de las redes sociales de los sirios y de los libaneses, sin suprimirlas. Un análisis de la reconversión a partir de 1950 será abordado en otro trabajo.

\footnotetext{
${ }^{30}$ Moldes y Entraigas describieron, a partir de datos censales, el proceso de migración en Río Negro de las zonas rurales a los centros urbanos durante el período. Moldes, Beatriz y Entraigas, Jorge. "La población rionegrina durante la época territorial. Un aporte al conocimiento de las principales características demográficas: 18841955", en Ruffini, Martha y Masera, Ricardo (coords.), Horizontes en perspectiva. Contribuciones para la Historia de Río Negro 1884-1955 (Viedma: Fundación Ameghino, 2007), pp. 71-122.
} 
Cuadro 1. Comercios registrados en la ciudad de Valcheta entre 1953 y 1955, resaltados los propietarios de origen sirio o libanés.

\begin{tabular}{|c|c|c|c|}
\hline Propietario & Rubro & Escala comercial & $\begin{array}{l}\text { Capital en } \\
\text { pesos }\end{array}$ \\
\hline Asconape & Lechería & minorista & 90.000 \\
\hline Bani de Chico Rosa & Verdulería & minorista & 2.000 \\
\hline Casa García & Almacén & mayorista & 335.000 \\
\hline Chibli Ali Salin & Tienda & minorista & 10.000 \\
\hline Contenti Ángel & Almacén de ramos generales & minorista & 35.000 \\
\hline Dillerico José & Venta ambulante de pescado & minorista & 1.500 \\
\hline Gattoni Manuel & Zapatería & minorista & sin datos \\
\hline Giménez Mauricio & Almacén & minorista & 20.000 \\
\hline González Erasmil & Almacén & minorista & 12.000 \\
\hline Lorca y Cia & Proveeduría & mayorista & 260.000 \\
\hline Mellado José Antonio & Bar y cine & minorista & 53.000 \\
\hline Milache Juan & Carnicería & minorista & 3.000 \\
\hline Missitti Prospero & Verdulería y despensa & minorista & 2.000 \\
\hline Morales Julio & Artículos comestibles & minorista & 22.000 \\
\hline Morón Eriberto & Peluquería y tienda & minorista & 1.100 \\
\hline Mortada Said & $\begin{array}{l}\text { Librería y artículos en } \\
\text { general }\end{array}$ & minorista & 20.000 \\
\hline Mussi Medhi & Almacén & minorista & 20.000 \\
\hline Mussi Saluh Hussain & Almacén y verdulería & minorista & 10.000 \\
\hline Rada Ismael & Carnicería y despensa & minorista & 50.000 \\
\hline Said Mortada & $\begin{array}{l}\text { Venta de cervezas y bebidas } \\
\text { gaseosas }\end{array}$ & minorista & 30.000 \\
\hline Scandroglio José & Panadería & minorista & 1.000 \\
\hline Seleme Elías & $\begin{array}{l}\text { Venta ambulante de frutas y } \\
\text { verduras }\end{array}$ & minorista & 30.000 \\
\hline Torres Olga & Pensión y bar & minorista & 5.000 \\
\hline Trujillo Ángela & Almacén & minorista & 5.000 \\
\hline Urcain Manuel & Artículos comestibles & minorista & 5.000 \\
\hline Vega Adrián & Verdulería & minorista & 20.000 \\
\hline Wucusich Emilio & Ferretería & minorista & 246.184 \\
\hline Yeber José & $\begin{array}{l}\text { Peluquería, perfumería, } \\
\text { cigarrería, golosinas }\end{array}$ & minorista & 10.000 \\
\hline Youssif Kemal Kamel & Almacén de ramos generales & minorista & 20.000 \\
\hline Zaher Tufic & Almacén & minorista & 30.000 \\
\hline
\end{tabular}

Fuente: elaborado a partir del Registro Único de Comercios de la Provincia de Río Negro (AHPRN). 


\section{Palabras finales}

En este trabajo logramos identificar a inmigrantes y a bolicheros sirios y libaneses, quienes pudieron reconstruir parcialmente sus redes familiares y comerciales, las cuales podían ser complementarias. En este sentido, consideramos significativo el desarrollo de grafos, mediante los cuales pudimos representar dichas redes sociales y sus articulaciones. A partir del análisis de estas redes dimos cuenta de la fuerte movilidad de los sirios y los libaneses por la región, en busca de potenciales mercados o con el objetivo de acceder a recursos estratégicos.

La aplicación de ARS combinado con la georreferenciación de desplazamientos e itinerarios, es una herramienta potente para la reconstrucción de la materialidad y la espacialidad de prácticas históricas. El ARS habilita la reconstrucción de las redes de relaciones de actores y espacios que han tendido a estudiarse en forma aislada, pero que sin embargo aparecen conectados, como estancieros y crianceros ocupantes de tierras fiscales, o los espacios rurales y pueblerinos en los que los comerciantes desarrollaron prácticas complementarias.

Las redes comerciales de los sirios y los libaneses demostraron la capacidad de convivir competitivamente en un mismo espacio. Los intereses económicos de diferentes sujetos o grupos tendían a no superponerse. En este sentido, pudimos reflejar el proceso de movilidad de los hijos de los bolicheros, quienes se trasladaban a otros parajes para no entrar en competencia con sus padres o como parte de la estrategia de expansión territorial de las redes de negocios familiares.

De nuestro análisis surge que los sirios y los libaneses no eran un colectivo homogéneo. Si bien es verdad que la mayoría fueron pequeños y medianos bolicheros, algunos fueron grandes comerciantes con una importante influencia política. Quizás la fuerte heterogeneidad socio-económica de los inmigrantes sirios y libaneses haya atentado contra la conformación de una organización étnica que aglutinara a este grupo. Recordemos que pese a la densa población de sirios y de libaneses en un territorio relativamente pequeño, la organización no se concretó sino hasta la segunda mitad de la década de $1950^{31}$.

Según las fuentes consultadas, estos comercios no tuvieron conflictos importantes con las grandes casas comerciales en la competencia por el mercado común. Inferimos que los comerciantes sirios y libaneses funcionaron como intermediarios comerciales y llegaron hasta donde las grandes casas comerciales no lo hacían, operando con eficacia en una geografía compleja.

\footnotetext{
${ }^{31}$ A partir de los aportes de los evaluadores del artículo, debemos mencionar que desde 1927 funcionó una Asociación Libanesa en la localidad de General Roca dentro de la Provincia de Río Negro, organización en la que muchos libaneses de la línea sur participaron, formando parte de dicho espacio de sociabilidad y solidaridad, lo que plantea otra cuestión a considerar a la hora de explicar la tardía creación de una asociación étnica para sirios y para libaneses en Valcheta.
} 
Por último, pudimos matizar el proceso de decadencia de los boliches: si bien se produjo una evidente crisis, las consecuencias pueden haber tendido a la reconversión comercial concentrándose en las ciudades, más que a la extinción, lo que nos indica una relativa flexibilidad y capacidad de readaptación a los cambios contextuales de las redes territoriales construidas durante la primera parte del siglo XX por los bolicheros sirios y libaneses a lo largo de la línea sur de Río Negro.

\section{Fuentes}

\section{Fuentes primarias}

\section{Archivos}

Archivo Histórico de la Provincia de Río Negro (AHPR), Registro Único de Comercios de la Provincia de Río Negro (1953-1955).

AHPR, Inspecciones de la Dirección General de Tierras en San Antonio y la Colonia Valcheta con sus zonas de influencia I y II (1904-1920).

AHPR, Informes de Policía del territorio de Río Negro.

Archivo Registro Civil Ciudad de Valcheta. (ARCC). Archivo del Museo Regional María Inés Koop de la Ciudad Valcheta.

\section{Fuentes secundarias}

\section{Libros}

Alsina, Juan. La inmigración en el primer siglo de la independencia. Buenos Aires: editado por Felipe S. Alsina, 1910.

Jong, Gerardo de. Introducción al método regional. Neuquén: Lipat-Universidad Nacional del Comahue, 2001.

Ramos, Ana. Los pliegues del linaje: memorias y politicas mapuches- tehuelches en contextos de desplazamientos. Buenos Aires: Eudeba, 2010.

Reynoso, Carlos. Redes sociales y complejidad: modelos interdisciplinarios en la gestión sostenible de la sociedad y la cultura. Buenos Aires: Editorial SB, 2011.

Salomón Tarquini, Claudia. Largas noches en La Pampa. Itinerarios y resistencias de la población indígena (1878-1976). Buenos Aires: Prometeo, 2010.

Vezub, Julio. Valentín Saygüeque y la gobernación Indígena de las Manzanas, Poder y etnicidad en la Patagonia septentrional (1860-1881). Buenos Aires: Prometeo, 2009. 
Redes sociales y territorialidad bolichera de sirios y libaneses en el sudeste de Río Negro,...

\section{Capítulos de libros}

Benedetti, Alejandro. "Territorio: concepto integrador de la geografía contemporánea", en Souto, Patricia (coord.), Territorio, lugar, paisaje. Prácticas y conceptos básicos en geografía. Buenos Aires: Editorial de la Facultad de Filosofía y letras de la Universidad de Buenos Aires, 2011.

Debattista, Susana y Destéffaniz, Carolina. "El difícil arte de juzgar y ser justo", en Gentile, María Beatriz; Rafart, Carlos Gabriel y Bohoslavsky, Ernesto (comps.), Historias de sangre, locura y amor, Neuquén (1900-1950). General Roca: Publifadecs, 2000.

Favaro, Orietta e Iuorno, Graciela. "Entre territorio y provincia. Libaneses y sirios, comercio y política en Neuquén”, en Favaro, Orietta (ed.), Neuquén, la construcción de un orden estatal. Neuquén: Centro de Estudios Históricos de Estado, Política y Cultura, 1999.

Iuorno, Graciela. "La provincialización de Río Negro. Interregno y conflictos de intereses nacionales y locales", en Ruffini, Martha y Masera, Ricardo (coords.), Horizontes en perspectiva. Contribuciones para la Historia de Río Negro 1884-1955. Viedma: Fundación Ameghino, 2007.

Miceli, Jorge y Guerrero, Sergio. "Redes libres de escala y su uso en el análisis de datos etnográficos: el caso de la comunidad tehuelche del Chalía", en Díaz, Diego, et al. (comp.), Exploraciones en antropología y complejidad. Buenos Aires: Editorial SB, 2007.

Moldes, Beatriz y Entraigas, Jorge. "La población rionegrina durante la época territorial. Un aporte al conocimiento de las principales características demográficas: 1884-1955", en Ruffini, Martha y Masera, Ricardo (coords.), Horizontes en perspectiva. Contribuciones para la Historia de Río Negro 1884-1955. Viedma: Fundación Ameghino, 2007.

Suárez, Graciela. "La Justicia Letrada: su organización y funcionamiento en el Territorio Nacional de Río Negro, 1879-1915”, en Ruffini, Martha y Masera, Ricardo (coord.), Horizontes en perspectiva. Contribuciones para la Historia de Río Negro 1884-1955. Viedma: Fundación Ameghino, 2007.

Sourrouille, Marcos y Vezub, Julio. “¿Largos peregrinajes en el vacío? Indagaciones sobre desplazamientos de indígenas y colonos en la Patagonia central", en Vessuri, Hebe y Bocco, Gerardo (coord.), Conocimiento, paisaje y territorio. Procesos de cambio individual y colectivo. Río Gallegos: UNAM, UNPA, UNRN, CENPATCONICET, 2014.

\section{Artículos en revistas}

Bandieri, Susana. "Entre lo micro y lo macro: la historia regional. Síntesis de un experiencia", en Entrepasados, n. ${ }^{\circ} 11,1996$. 
Boschín, María Teresa y del Castillo Bernal, Florencia. "El Yamnago: del registro histórico al registro arqueológico", en Revista Española de Antropología Americana, n. ${ }^{\text {o } 36,2005 . ~}$

Boschín, María Teresa y Andrade, Analía. "Poblamiento de Patagonia Septentrional Argentina durante el holoceno tardío: paleoambientes e imperativos sociales", en Zephyrus, n. ${ }^{\circ}$ 68, 2011.

Chávez, Matías. "Sujetos y espacios marginales en la narrativa de Asencio Abeijón (Chubut, 1900-1920)", en Atek Na [En la tierra], n. ${ }^{\circ}$ 3, 2013.

Favaro, Orietta e Iuorno, Graciela. "Libaneses y sirios. Actividades comercial y participación en el espacio público”, en Entrepasados, n. ${ }^{\circ}$ 17, 1999.

Ibarra, Horacio y Hernández, Carlos. "Huellas, picadas, carros, boliches. Poblamiento y comunicaciones en la Patagonia vieja", en VI Congreso de Historia Social y Política de la Patagonia argentino-chilena, 2007.

Salomón Tarquini, Claudia; Vezub, Julio y Escolar, Diego. "Cartografía de redes sociales en pampa y Patagonia: sistematización de correspondencia de líderes indígenas mediante bases de datos inteligentes", en Primer Congreso Internacional Las poblaciones indígenas de América Latina, Siglos XIX-XXI. Avances, perspectivas y retos, 2013.

Vezub, Julio. "Lenguas, territorialidad y etnicidad en la correspondencia de Valentín Saygüeque hacia 1880", en Intersecciones en Antropología, n. ${ }^{\text {o } 7,2006 .}$ 